\title{
Archives de musique arabe. Congrès du Caire, 1932
}

\section{Pierre Bois}

\section{OpenEdition \\ Journals}

Édition électronique

URL : http://journals.openedition.org/ethnomusicologie/2411

ISSN : 2235-7688

Éditeur

ADEM - Ateliers d'ethnomusicologie

Édition imprimée

Date de publication : 1 janvier 1990

Pagination : 249-253

ISBN : 2-8257-0423-7

ISSN : $1662-372 X$

Référence électronique

Pierre Bois, «Archives de musique arabe. Congrès du Caire, 1932 ». Cahiers d'ethnomusicologie [En ligne], 3 | 1990, mis en ligne le 15 octobre 2011, consulté le 05 mai 2019. URL : http:// journals.openedition.org/ethnomusicologie/2411

Ce document a été généré automatiquement le 5 mai 2019.

Article L.111-1 du Code de la propriété intellectuelle. 


\title{
Archives de musique arabe. Congrès du Caire, 1932
}

\author{
Pierre Bois
}

\section{RÉFÉRENCE}

Archives de la musique arabe - vol. 1 (1908-1920 env.). Textes de présentation : Bernard Moussali et Christian Poché. Un CD Ocora/Radio France et Institut du Monde arabe, C 558 678, 1987.

Maroc : musique classique. Congrès du Caire 1932. Cheikh Mohamed Chouika \& Omar Jaïdi (1932). Textes de présentation : A. Hachlef. Un CD Artistes Arabes Associés, AAA 006, 1989. [Anthologie de la musique arabe]. Congrès du Caire 1932 - vol. 1 et 2 (1932). Textes de présentation : Bernard Moussali et Christian Poché. Deux CD Bibliothèque nationale/Institut du Monde Arabe, Paris, APN 88-9/10, 1988.

Archives de la musique arabe - vol. 1 (1908-1920 env.). Textes de présentation : Bernard Moussali et Christian Poché. Un CD Ocora/Radio France et Institut du Monde arabe, C 558 678, 1987.

Maroc : musique classique. Congrès du Caire 1932. Cheikh Mohamed Chouika \& Omar Jaïdi (1932). Textes de présentation : A. Hachlef. Un CD Artistes Arabes Associés, AAA 006, 1989. [Anthologie de la musique arabe].

Congrès du Caire 1932 - vol. 1 et 2 (1932). Textes de présentation : Bernard Moussali et Christian Poché. Deux CD Bibliothèque nationale/Institut du Monde Arabe, Paris, APN 88-9/10, 1988.

1 Les musiciens arabes, ne sachant s'ils doivent se replier sur la tradition ou faire table rase du passé, ne compter que sur leurs capacités créatrices ou emprunter des modèles régénérateurs à l'Occident, traversent actuellement une grave crise d'identité qui se traduit par le triomphe de la chansonnette, la raréfaction d'œuvres nouvelles, le manque 
de vocations des jeunes, et l'absence de crédit(s) de la part des pouvoirs publics des pays concernés, particulièrement au Machrek.

2 Dans une telle situation d'incertitude et de doute, les références constantes au Congrès historique de la Musique arabe du Caire en 1932, font figure de rites propitiatoires. Autant de colloques savants, d'assemblées de notables et, depuis deux ans, de publications d'archives phonographiques, qui se veulent les contre-chants de l'illustre événement. En son temps pourtant, celui-ci posait déjà les problèmes de la création, de l'enseignement et des statuts de la musique arabe, si cruciaux en cette fin de siècle.

3 Certes, cette époque fut glorieuse pour la musique arabe ; le disque, qui permit d'étendre la notoriété des grandes voix égyptiennes, syriennes, irakiennes et libanaises au-delà des frontières, y eut sa part et participa directement à la suprématie de la musique égyptienne dans tous les pays arabes.

4 Introduite en 1904, l'industrie balbutiante du 78 tours connut un essor rapide, notamment au Caire et à Alexandrie ${ }^{1}$; en quelques années, les compagnies étrangères (Gramophone, Polyphone, Odéon, Columbia) se virent supplantées par des firmes locales basées en Égypte, mais aussi à Alep, Bagdad et Beyrouth.

5 Cette époque des pionniers est remarquablement présentée dans un CD co-produit par l'Institut du Monde arabe et Ocora/Radio France: Archives de la musique arabe, volume 1, consacré à plusieurs grands musiciens du Machrek de 1908 à 1920.

6 Malgré les défauts inhérents à une technologie rudimentaire (la prise de son électrique n'existait pas encore), ce disque offre un panorama de formes musicales savantes, considérées alors comme les seules dignes d'être gravées, à savoir: le taqsim (improvisation instrumentale), la qasida (chant improvisé sur un poème classique), le mawwal (chant improvisé sur un poème dialectal), et le dawr (chant à refrain en dialecte égyptien) dont le succès à la fin du XIX ${ }^{\mathrm{e}}$ siècle contribua à l'hégémonie de la musique égyptienne. Ces pièces sont exécutées par quelques-uns des grands noms de l'époque, prédécesseurs de Sayyed ad-Darwish et d'Umm Kulthūm, chanteurs formés à l'école des confréries soufies comme il était de tradition depuis plusieurs siècles dans l'Empire ottoman. A travers quatre exemples de qasida, on peut apprécier les grandes différences de styles vocaux qui opposent les maniérismes ornementaux du Syrien Muhamad al-'Ashiq à la limpidité d'expression et la réserve du célèbre Cairote Yusuf Khafaji alManyalawi, chanteur à la cour khédiviale, ou encore la voix intérieure mais pleine de passion contenue de Salama Higazi, créateur de la musique de scène en Égypte, à la déclamation disloquée et émaillée de subtils points de suspension d"Abd al-Hayy Hilmī...

7 On appréciera par ailleurs le gros travail d'analyse et de documentation de Christian Poché et Bernard Moussali, qui nous permet d'avoir une vision à la fois synthétique et détaillée de la pratique musicale arabe du Machrek au début de ce siècle.

8 Le Club du disque arabe, de son côté, consacre un CD entier aux enregistrements de l'orchestre envoyé au Congrès du Caire par le Roi du Maroc, sous la direction du Cheikh Mohamed Chouika et d'Omar Jaïdi. Il s'agit d'une vingtaine de pièces vocales et instrumentales sélectionnées parmi l'immense corpus des onze nūbā qui constituent le répertoire du āla arabo-andalou du Maroc.

9 La beauté de l'interprétation, l'enthousiasme plein de retenue des chanteursinstrumentistes, l'homogénéité sonore de l'ensemble, prouvent une fois de plus l'inanité des pupitres d'orchestres symphoniques des ensembles arabo-andalous modernes. 


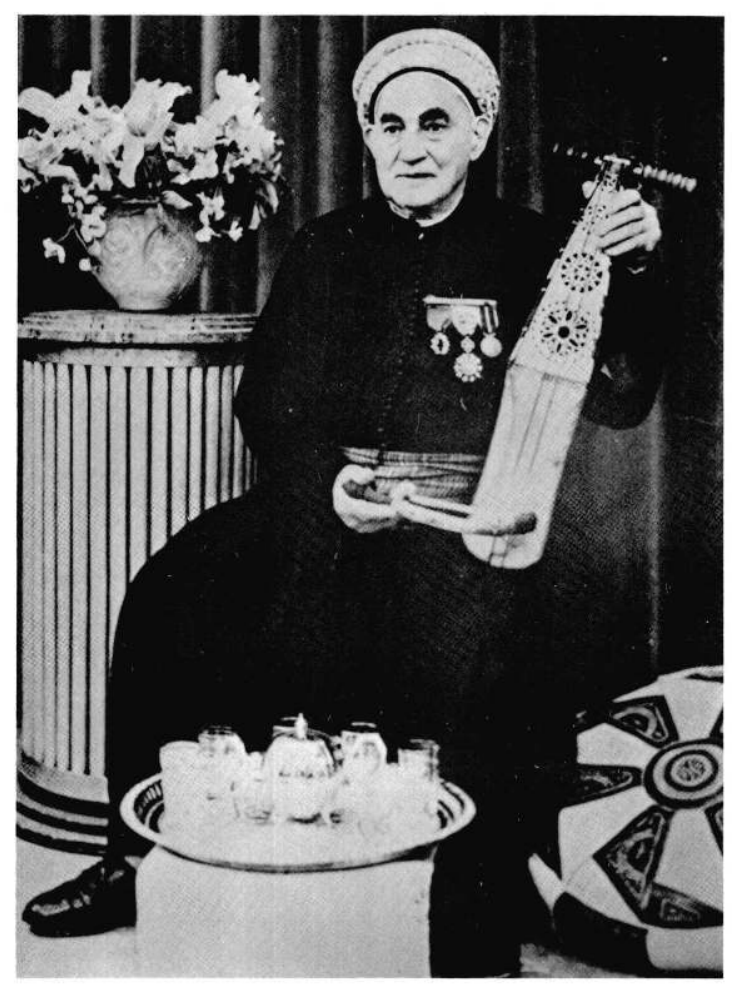

Le Shaykh Al-'Arabi ben Sādi, chef de l'ensemble de Tlemcen (Algérie), jouant du rabāb. Photo ancienne.

Plus critiquables sont le texte de présentation et le mode de montage du disque, qui tendent à égarer l'auditeur peu familiarisé avec ce répertoire. Si le livret nous présente sur plusieurs colonnes l'historique des $n \bar{u} b \bar{a}$, il omet en revanche de les décrire ${ }^{2}$ et surtout ne référencie les pièces publiées que par les incipits des poèmes sans préciser de quelles $n \bar{u} b \bar{a}$ elles sont extraites, rendant ainsi toute comparaison impossible avec des versions plus récentes. Quant au montage, il a été conçu comme un mouvement de nūbā (mīzān), c'est-à-dire une succession de pièces vocales collées les unes aux autres et précédées de deux pièces instrumentales introductives. Mais, du fait de la diversité d'origine des morceaux, cette construction conduit souvent à des incohérences modales. Dans une publication d'archives, rien ne s'opposait à une présentation plus scientifique et détaillée.

11 Il serait illusoire de prétendre présenter en quelques lignes le monumental travail produit par l'Institut du Monde Arabe et la Phonothèque Nationale et consacré au Congrès du Caire : deux disques compacts et un livret trilingue (français, anglais, arabe) de 200 pages rédigé par Christian Poché et Bernard Moussali.

Organisé à la demande du roi Fouad d'Égypte, le Congrès du Caire, qui se déroula du 28 mars au 3 avril 1932, avait pour objet de faire le point sur la musique arabe: statuts, enseignement, orientations créatrices. Le problème que se posaient les théoriciens arabes en cette fin de période de nahda (renaissance intellectuelle), était de "sortir » la musique arabe de la stagnation, et grâce aux progrès apportés par une théorie unificatrice et un enseignement de type occidental, l'amener vers sa vraie place dans la «musique universelle» (i.e. occidentale). Il fallait donc la doter d'une théorie explicite qui rende compte de manière unifiée des diverses tendances régionales et, tout d'abord, se mettre d'accord sur les problèmes de division de l'échelle. Cela fait, certains théoriciens prônaient une réorientation de la création vers un courant original comparable à l'œuvre 
des Russes du Groupe des Cinq. Curieusement, les objections à une telle tentative vinrent des participants européens, notamment Curt Sachs et Béla Bartok, qui craignaient de voir la musique arabe s'enfermer dans un courant normalisateur. Et c'est dans ce sens que Bartók, chargé de la commission d'enregistrement des groupes de musiciens invités au Congrès, refusa d'entériner la "modernité » des groupes envoyés par le Liban et la Syrie. Ainsi, sur les 360 faces de 78 tours enregistrées par la firme Gramophone, et dont la Phonothèque Nationale est l'un des rares organismes à avoir conservé les 335 faces épargnées par le temps, ne figurent que des groupes irakiens, égyptiens, marocains, tunisiens et algériens.

13 Le premier disque présente tout d'abord la tradition savante de Bagdad avec la voix fabuleuse du chanteur et poète Muhammad al-Qubbanji, et la tradition populaire citadine du Caire.

Chanteur de la nouvelle génération, Qubbanji adopta une démarche d'ouverture vers les autres pays arabes, en adjoignant aux instruments du traditionnel tshalghi baghdadi le luth 'ūd et la cithare à cordes pincées qanūn du takht proche-oriental. Il fit également un large usage de modes d'origine ottomane et syro-égyptienne. On l'entend ici dans une abudhiyya suivie d'un long maqam d'une vingtaine de minutes, et d'une pasta, trois formes spécifiquement irakiennes, auxquelles succèdent trois improvisations instrumentales (taqasim), au santūr, à la jawza et au 'ūd.

différent de la tradition arabe, à savoir divers aspects de la pratique populaire urbaine, avec le chanteur de mawwal Muhammad al-'Arabi (qui fut le maitre du Druze syrien Farid al-Atrash), un ensemble d'aimées, musiciennes citadines professionnelles si bien décrites dans les romans de Mahfuz, et dont le répertoire initialement destiné à un public féminin comprenait des chants de mariage, de circoncision, de fêtes et de funérailles, un ensemble de clarinettes doubles miz-mar et de tambours accompagnant les fêtes officielles, un groupe de bédouins du Fayyoum, et des chants d'exorcisme zār dont les origines remontent probablement à la période pharaonique.

Le second disque est entièrement consacré aux musiques citadines du Maghreb : tradition de Tlemcen, exempte d'influences proches-orientales, avec des extraits de diverses nūbu, tradition savante de Fès dont les pièces ne recoupent heureusement pas celles qui ont été sélectionnées dans le disque du Club du Disque Arabe, et enfin la musique citadine de Tunis dont le style indique bien la confluence des cultures musicales du Machrek et du Maghreb.

\section{NOTES}

1. Voir à ce sujet Ali Jihad Racy, «Record industry and Egyptian traditional music : 1904-1932 ». Ethnomusicology 20(1), 1976 : 23-48 [Ndlr].

2. Al-āla se base actuellement sur onze modes (tubu) principaux. Sur chacun de ces modes a été composé une nūbā, long cycle vocal et instrumental. Chaque nūbā se compose de cinq mouvements ou mizān déterminés par des structure rythmiques spécifiques et discrètes qui se 
modifient progressivement en fonction d'une accélération en trois phases (lent - moyen - vif) ; chaque mīzān débute généralement par un prélude instrumental de rythme libre (bughya), suivi d'une pièce instrumentale mesurée (tawshiya), qui introduisent la suite des pièces vocales (san'a) exécutées par le chœur des instrumentistes dans le rythme propre au mīzān: basit, qaym wa nusf, btayhi, darj, quddam. Les san'a sont des poèmes de 2, 3, 5 ou 7 vers entrecoupés de ritournelles instrumentales. Il est rare qu'une nûbā soit exécutée dans son intégralité ; le plus souvent, les ensembles choississent un mīzān, ou font un montage d'extraits de plusieurs mìzān, mais ce choix se situe toujours à l'intérieur d'une seule et même nūbā, afin de conserver l'expressivité propre au mode sur lequel elle a été composée (sentiment modal). 